\title{
Optimization of ion implantation damage annealing by means of high-resolution X-ray diffraction
}

\author{
J. G. E. Klappe and I. Bársony* \\ MESA Research Institute, University of Twente, P.O. Box 217, 7500 AE Enschede (Netherlands)
}

J. R. Liefting

FOM Institute for Atomic and Molecular Physics, Kruislaan 407, 1098 SJ Amsterdam (Netherlands)

T. W. Ryan

Philips Analytical, Lelyweg 1, 7602 EA Almelo (Netherlands)

(Received March 12, 1993; accepted April 29, 1993)

\begin{abstract}
High-resolution X-ray diffraction (HR-XRD) was investigated as a possible technique for the qualitative analysis of damage annealing of low-dose, high-energy implanted (001) silicon, implanted with dopants smaller than the host atom. The choice of proper Bragg reflection for the rocking-curve measurements is shown to be of crucial importance. The graphic construction of the Ewald sphere is a useful aid for this purpose. As the in-plane lattice constant is confined by the underlying substrate, a change occurs in the perpendicular direction only. Therefore the (026) 1 reflection appears to be the most suitable for the detection of changes in lattice constant caused by implantation damage. Qualitative analysis of rocking curves of P- and B-implanted Si samples was compared with electrical measurements and cross-section transmission electron micrographs. It could be established that the minimum implantation doses of $\mathrm{P}$ and $\mathrm{B}$ at energies ranging from 0.5 to $1.5 \mathrm{MeV}$, for which HR-XRD is sensitive enough, are about $1.5 \times 10^{14} \mathrm{~cm}^{-2}$ and $5 \times 10^{13} \mathrm{~cm}^{-2}$ respectively. The minimum peak temperature needed for complete damage anneal by transient-rapid thermal annealing was about $1400 \mathrm{~K}$ for all doses considered.
\end{abstract}

\section{Introduction}

High-energy ion-implantation is an increasingly favored technique for retrograde profile buried-layer formation in advanced silicon processing [1]. Crystal damage introduced by this doping technique can be removed by annealing. Short-time, high-temperature treatments, such as transient-rapid thermal annealing (T-RTA), are compatible with the requirement of low thermal budget processing $[2,3]$. Analysis of doping and damage profiles of implanted silicon has been performed using a number of techniques, such as (cross-section) transmission electron microscopy ((X)TEM) [4-9], secondary ion mass spectrometry (SIMS) [10-13], Rutherford backscattering spectrometry (RBS) [14], Secco etching [15] $C-t$ measurements $[16,17]$ or $C-V$ measurements [16]. Most of these techniques are destructive or require special sample preparation. X-ray diffraction (XRD) is a non-destructive technique which is widely used in the study of changes in lattice constant and damage in dopant diffusion [18], ion-implanted

*Present address: KFKI Research Institute for Materials Science, P.O. Box 49, 1525 Budapest, Hungary. crystals [19-22], epitaxial layers [23] and compound semiconductors [19, 24]. Most of these papers deal with $\mathrm{X}$-ray measurements of high-dose (greater than or equal to $10^{15}$ ions $\mathrm{cm}^{-2}$ ), low-energy (less than or equal to $200 \mathrm{keV}$ ) implanted silicon. There are only a few reports on measurements of low-dose implanted silicon [25]. In the case of low-dose, high-energy implantations, the change in lattice constant of the crystal is very small and located at a relatively large distance from the surface, imposing high requirements on the resolution of the measuring equipment. The choice of Bragg reflection which is most sensitive to the lattice constant changes becomes crucial.

This paper describes the qualitative analysis of the measured rocking curves (rcs). The limitations of this measurement technique with regard to the smallest detectable implanted dose are discussed. Furthermore, the use of high-resolution (HR) XRD is presented for optimizing annealing of low-dose implants in the case of dopants with a smaller atomic size than the host atom. Complementary techniques such as XTEM and electrical measurements were used for comparison.

A quantitative analysis, involving the determination of strain as a function of depth by means of the dynamical theory of XRD, will be the subject of a separate paper. 


\section{Lattice disorder in ion-implanted silicon}

Dopants implanted into Si come to rest by electronic and nuclear stopping mechanisms. The ions collide with nuclei as well as with electrons of the atoms of the accommodating lattice. Electronic stopping involves a small energy loss per distance and a small deflection of the incoming ion. Nuclear collisions, however, can cause considerable damage to the lattice. The host atoms are displaced by the collisions when a threshold energy of $E_{\mathrm{d}}=15.8 \mathrm{eV}$ [26] is transferred. In the case of an energy transfer of more than $2 E_{\mathrm{d}}$ the displaced atom itself can displace more atoms, which results in a cascade of atomic collisions. As a consequence a distribution of point defects, vacancies, interstitials and amorphous regions is generated during implantation. Light elements such as B lose energy mainly in electrical collisions; therefore they cause less damage than heavier implants stopped mainly by nuclear scattering [28].

The rate of energy loss, the disorder introduced by the implantation step, i.e. the number of displaced atoms, depends on the dose and energy of the implanted element as well as on the substrate temperature. The very moderate ion-current density of $0.1 \mu \mathrm{A} \mathrm{cm}-2$ used in our experiments excluded the possibility of in situ damage annealing and the substrate remained at room temperature throughout the implantation steps. In view of our low-dose megaelectronvolt $\mathrm{B}$ and $\mathrm{P}$ implants, in most cases we cannot expect amorphization. Since the purpose of any implantation step is to introduce a controlled number of electrically active dopants, the lattice disorder around the incorporated dopant ions, which may be a region of point defects, amorphous or contain extended defects, must be removed. Otherwise the abundance of electron and hole traps and recombination centers present would dominate the electrical performance of the implanted layer. The restoration of lattice perfection is achieved by post-implantation damage annealing. This step in nonamorphized structures has a combined function. The interstitial doping atoms have to be moved to substitutional lattice sites to obtain electrical activity and the crystal damage has to be eliminated. The primary defects usually coalesce to form secondary defects, i.e. dislocation loops, which grow to extended defects. These propagate to interfaces, which are natural sinks for defects. The elimination of point defects at an early phase of damage annealing is essential to minimize the chance of interaction between point defects and dopant atoms. Their complexes, e.g. the B-vacancy complex, have, owing to their reduced activation energy, a much faster diffusivity than the dopant atoms. This leads to the undesirable effect of point defect-enhanced diffusion, limiting the formation of shallow, abrupt $p-n$ junctions by $B$ implantation.
Any high-temperature treatment contributes to the redistribution of the implanted doping profile, therefore the post-implantation anneal has to be restricted to a minimum thermal budget. The reduction of this budget is accomplished conventionally by lowering the annealing furnace temperature to $1100-1300 \mathrm{~K}$. This, however, cannot prevent enhancement of the diffusion caused by the damage. Furthermore, the high activation energy of $\mathrm{Si}$ self-diffusion, $E_{\mathrm{a}}=5 \mathrm{eV}[27,28]$, which is required for point-defect annihilation, necessitates the use of higher annealing temperatures. A viable option is rapid thermal annealing (RTA), or transient (T-) RTA, with a much shorter execution time.

\section{High-resolution X-ray diffraction for lattice disorder studies}

HR-XRD is sensitive to the change $\Delta a$ in the lattice constant $a$, or to the strain $\Delta a / a$. The strain in the crystal is positive when intersitials are present, and negative when the dopant atoms smaller than the host atom ( $\mathrm{P}$ and $\mathrm{B}$ in $\mathrm{Si}$ ) reside at substitutional lattice sites. Qualitative analysis of the crystalline perfection of implanted substrates is based on this effect. The strain introduced by low-mass, low-dose implants is very small. When all the implanted ions are at substitutional lattice sites the maximum strain lies at the depth of the peak of the doping concentration profile. Both the maximum dopant concentration $C_{\max }$ and its depth depend on the implantation energy. The maximum strain $(\Delta a / a)_{\max }$ can be calculated using the lattice contraction coefficients [29] (Table 1).

The theoretical sensitivity limit of XRD is $10^{-5}$ [30]. Accordingly, the sensitivity limit for substitutional dopants in the case of $1.5 \mathrm{MeV}$ implanted $P$ is about $2 \times 10^{14} \mathrm{~cm}^{-2}$ and in the case of $1 \mathrm{MeV}$ implanted $B$ it is about $8 \times 10^{13} \mathrm{~cm}^{-2}$. However, the strain caused by interstitials as a consequence of ion-implantation damage is about an order of magnitude higher. The strain margin between the as-implanted and the restored crystal leaves enough room for trend analysis.

The small strain imposes extreme requirements on the analytical equipment, therefore only HR-XRD is a

TABLE 1. Maximum change in lattice constant $(\Delta a / a)_{\max }$ for various implanted doses of $1500 \mathrm{keV} \mathrm{P}$ and $1000 \mathrm{keV} \mathrm{B}$

\begin{tabular}{llrr}
\hline Ion & Dose $\left(\mathrm{cm}^{-2}\right)$ & $C_{\max }\left(\mathrm{cm}^{-3}\right)$ & $(\Delta a / a)_{\max }$ \\
\hline P & $5 \times 10^{13}$ & $1.11 \times 10^{18}$ & $2 \times 10^{-6}$ \\
P & $1 \times 10^{14}$ & $2.2 \times 10^{18}$ & $4 \times 10^{-6}$ \\
P & $5 \times 10^{14}$ & $1.11 \times 10^{19}$ & $2 \times 10^{-5}$ \\
B & $5 \times 10^{13}$ & $1.6 \times 10^{18}$ & $7.2 \times 10^{-6}$ \\
B & $1 \times 10^{14}$ & $3.2 \times 10^{18}$ & $1.44 \times 10^{-5}$ \\
B & $5 \times 10^{14}$ & $1.6 \times 10^{19}$ & $7.2 \times 10^{-5}$ \\
\hline
\end{tabular}


viable option. The Bartels monochromator [30] with four $\mathrm{Ge}$ crystals used in the study has two possible settings. The (220) and the (440) settings offer a combination of a higher X-ray intensity with a relatively low resolution, and a lower intensity with a high resolution respectively. Many details of the curve are lost in the (220) setting, rendering it unacceptable for the analysis of low implanted doses. Therefore the (440) setting was used for the measurements.

Appropriate Bragg reflection selection is another prerequisite for the measurement of small changes in the lattice constant. Fukuhara and Takano [31] presented three criteria for proper selection of the reflection.

(1) The Bragg angle should be near $45^{\circ}$ in order to avoid the complication arising from mixed polarization states.

(2) The extinction distance $l$ should be reduced by selecting an asymmetric Bragg reflection.

(3) The following expression should be maximized for optimum sensitivity of the rc to the strain in the crystal:

$\left|\frac{\delta \theta_{0}}{\delta \varepsilon}\right| \frac{1}{\Delta \theta}=\frac{2 \cos \alpha \sin \theta_{\mathrm{B}}\left[\sin \left(\theta_{\mathrm{B}}+\alpha\right) \sin \left(\theta_{\mathrm{B}}-\alpha\right)\right]^{1 / 2}}{\left|\varphi_{\mathrm{h}}\right|}$

where $\Delta \theta$ is the half-width of the Bragg peak, $\theta_{\mathbf{B}}$ the Bragg angle, $\theta_{0}$ the glancing angle, $\alpha$ the angle between the reflecting lattice plane and the wafer surface and $\varphi_{\mathrm{h}}$ the relevant Fourier coefficient of the polarizability multiplied by $4 \pi$. In ref. 31 this resulted in the choice of the (422) $)_{1}$ reflection (the 1 indicates that the low-angle of incidence of $8.75^{\circ}$ was used). When the sample is bent, such a small angle of incidence results in peak broadening. In integrated circuit processing most of the wafers contain more or less warpage owing to thermomechanical stresses. The stress can be caused by the difference in thermal expansion coefficients of the deposited or grown layers on top of the wafer, or by strong thermal gradients developing across the specimen as a result of process inhomogeneities. Should a threshold value, the so-called yield stress, at a given process temperature be exceeded [32], the wafer experiences plastic deformation. Note that rapid thermal pro- cessing (RTP) is one of the most critical operations in this respect, especially in transient applications. Fast ramping enhances the inherent temperature drop at the edge of the processed wafer with reference to the temperature at the center, thereby limiting the effective time-temperature range of safe transient processing free of slip dislocation formation.

Also, improper mounting on the wafer stage of the diffractometer can cause mechanical stress. Sometimes unacceptable peak broadening was encountered with the (422), reflection. Therefore, in our opinion, a fourth requirement should be added to those in ref. 31: the wafers analyzed should be flat, otherwise the (422) reflection is not usable.

A more illustrative method than the abstract formula (1) for proper selection of the reflection is the Ewald sphere construction, as drawn in Fig. 1. Depth dependent changes in lattice constant and damage in the crystal cause an elongation of the reciprocal lattice point in all directions, as indicated schematically for the (004) reciprocal lattice point in Fig. 1(a). When an rc measurement is performed, the reciprocal lattice is rotated around the origin $O$ and the reciprocal lattice point (004) passes through the Ewald sphere. The measured intensity is the integrated intensity of the intersection of the Ewald sphere and the reciprocal lattice point.

(004) is not the most sensitive reflection for strain in the perpendicular direction. Maximum sensitivity is expected with a reflection where the direction of elongation is perpendicular to the surface of the Ewald sphere, according to the condition $2 \theta-\omega=90^{\circ}$. The (422), reflection satisfies this condition well, as $2 \theta-\omega=79.25^{\circ}$. However, this reflection has a low angle of incidence, causing peak broadening as explained previously. Therefore, we chose the $(026)_{1}$ reflection with $2 \theta-\omega=$ $127.5^{\circ}-45.3^{\circ}=82.2^{\circ}$. This reflection is constructed in Fig. 1(b). The sensitivity for the perpendicular strain is better than for the (422), reflection. The extinction depth $l$, however, is worse in this case, thereby violating the second requirement. Nevertheless, this reflection proved to be a better practical choice for our measurements, as

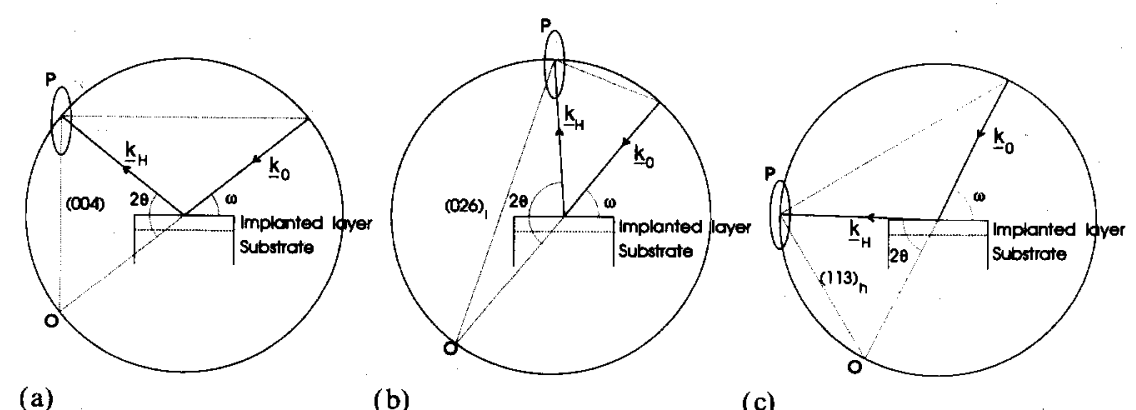

(a)

(b)

(c)

Fig. 1. Ewald-sphere construction for the symmetrical (004) reflection (a), and the asymmetrical (026) (b) and (113) $)_{\mathrm{h}}(\mathrm{c})$ reflections. 


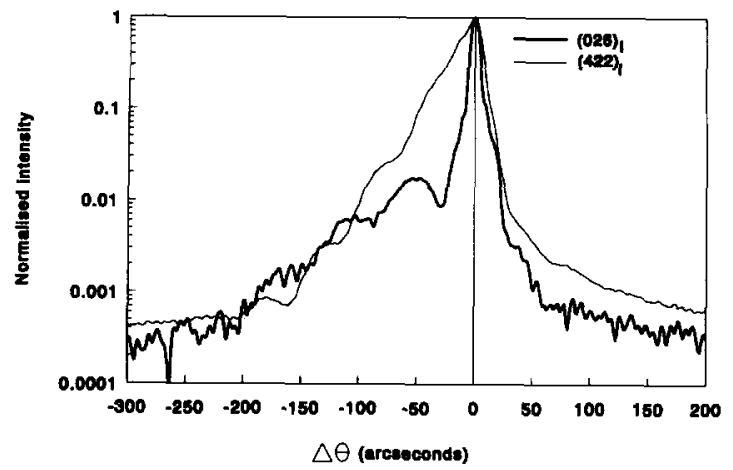

Fig. 2. Comparison between the (026), and (422), reflections of $P$ implanted $\mathrm{Si}\left(5 \times 10^{14} \mathrm{~cm}^{-2}, 0.5 \mathrm{MeV}\right)$.

the low doses implanted at high energy cause very small strain. Figure 2 shows a comparison between the (422), and the $(026)_{1}$ (normalized) rcs for P-implanted $\mathrm{Si}$, $5 \times 10^{14} \mathrm{~cm}^{-2}$ at $0.5 \mathrm{MeV}$. The separation of the main peak and the first fringe on the left side is very unclear in the (422) $)_{1}$ reflection, but is much better with the (026) reflection. The deconvolution is a problem for the strain profile determination with computer simulations.

The optimum condition for maximum sensitivity to the in-plane elongation of the reciprocal lattice points is $2 \theta-\omega=0$, i.e. the in-plane direction lies perpendicular to the surface of the Ewald sphere, as shown in Fig. 1(c). The (113) $)_{\mathrm{h}}$ reflection ( $\mathrm{h}$ is the high angle of incidence) is a good choice, with $2 \theta-\omega=56.1^{\circ}-$ $53.3^{\circ}=2.8^{\circ}$. For an extinction length of $10 \mu \mathrm{m}(\mathrm{Cu} \mathrm{K} \alpha)$, the depth from which the X-rays are diffracted is limited to about $0.5 \mu \mathrm{m}$ by the very small exit angle of $2.8^{\circ}$. HR-XRD analysis is thus restricted to the study of in-plane strain close to the sample surface. As an alternative, high-intensity synchrotron radiation provides much larger extinction lengths.

The measured res have a narrow peak with a full width at half-maximum (FWHM) varying between 6 and 12 arcsec. In all the rc representations, the $\Delta \theta$ axes are centered around this main peak originating from the substrate. The instrumental contribution to this FWHM is very small as a result of the use of a fourcrystal monochromator. The variance is mainly determined by the wafer bending, larger bending resulting in an increase in the FWHM.

Figure 3 illustrates the principle of qualitative rc analysis for post-implantation damage anneal optimization. The rcs of $5 \times 10^{4} \mathrm{~cm}^{-2}, 1 \mathrm{MeV}$ B-implanted $\mathrm{Si}$ wafers before and after annealing compared with that of virgin $\mathrm{Si}$ are shown. Fringes on the negative side indicate lattice expansion due to interstitial dopant and $\mathrm{Si}$ atoms. After treatment by RTA, which performs activation and lattice restoration simultaneously, the fringes left of the main peak diminish. On the positive

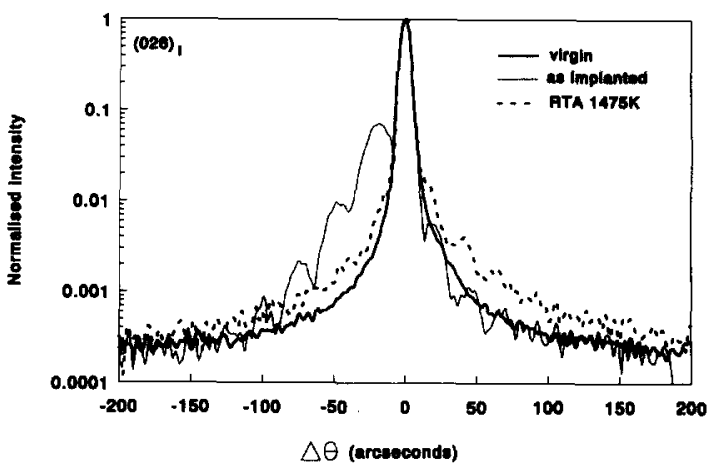

Fig. 3. The principle of damage anneal optimization by means of HR-XRD rocking curve analysis. Measured rocking curves of $5 \times 10^{14} \mathrm{~cm}^{-2}, 1 \mathrm{MeV}$ B-implanted $\mathrm{Si}$ before and after annealing, compared with unimplanted silicon.

side fringes appear indicating lattice contraction due to the smaller substitutional dopant atoms at $\mathrm{Si}$ lattice sites. The influence of intersitial atoms on the lattice constant is supposed to be much larger than that of substitutional dopants. It can therefore be assumed that no residual lattice damage, i.e. no primary defects, is visible in the rc of the annealed sample.

\section{Experimental details}

High-resistivity 6-10 $\Omega \mathrm{cm}$ (100) 3 inch silicon wafers were implanted through a screening thermal oxide layer of $80 \mathrm{~nm}$. Implantation of $P$ and $B$ was carried out at room temperature with energies ranging from 0.5 to $1.5 \mathrm{MeV}$, doses between $5 \times 10^{13}$ and $1 \times 10^{15} \mathrm{~cm}^{-2}$ and an ion-current density of $0.1 \mu \mathrm{A} \mathrm{cm}^{-2}$. Most of the samples were annealed in a Peak System LXU 35 RTP unit in $\mathrm{N}_{2}$ ambient. In order to shorten the effective anneal time we made use of a novel transient method (T-RTA) [2], by which isochronal anneals at peak temperatures $T_{\mathrm{p}}$ varying from 1075 to $1600 \mathrm{~K}$ could be reduced to a duration of $c a .300 \mathrm{~ms}$. Furnace anneals were also performed at 1200 and $1300 \mathrm{~K}$ for $30 \mathrm{~min}$ as a reference.

The rcs were measured with the Philips HR-XRD system MPD 1880/HR using a two-crystal, four-reflection (2C4R) Bartels [30] monochromator and a smallest angular step size of 0.9 arcsec. Triple-axis measurements were performed on a Philips Materials Research Diffractometer with the $2 \mathrm{C} 4 \mathrm{R}$ monochromator and a $1 \mathrm{C} 2 \mathrm{R}$ analyzer. All measurements were taken with $\mathrm{Cu} \mathrm{K} \alpha$ radiation.

XTEM with a Siemens Elmiskop 101 using an accelerating voltage of $100 \mathrm{kV}$ was applied in bright- and dark-field modes. 


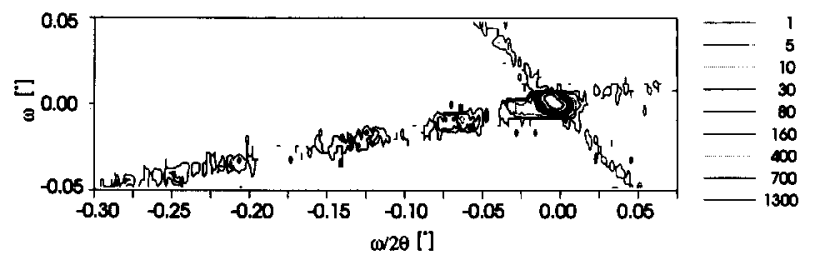

Fig. 4. Triple-axis measurement of the $(026)_{1}$ reciprocal lattice point in angular diffraction space $(\omega, \omega / 2 \theta)$ of $5 \times 10^{15} \mathrm{~cm}^{-2}, 45 \mathrm{keV}$ Bimplanted Si.

\section{Results and discussion}

In these experiments we followed the objective of determining the suitability of the HR-XRD technique for post-implantation anneal optimization in the case of low-dose, high-energy implants. We had to establish the limiting factors for the resolution and accuracy of this analytical technique for our purpose. The measurements focused on analysis of the effect of the following variables on the X-ray rcs for both dopants: implant energy, implant dose, anneal temperature, and anneal time.

First, the direction of the strain due to the ionimplantation was determined from the triple-axis measurement. Figure 4 shows the (026) reciprocal lattice point in $\omega-\omega / 2 \theta$ angular space after a $5 \times 10^{15} \mathrm{~cm}^{-2} \mathrm{~B}$ implantation at $45 \mathrm{keV}$. Two streaks are visible. The streak that makes an angle of $45^{\circ}$ with the axis is the analyzer streak, caused by the acceptance angle of the analyzer crystals. The other streak at an angle of about $10^{\circ}$ with the $\omega / 2 \theta$ axis is due to the increased lattice parameter after the ion-implantation. Using the formulae given by Iida and Kohra [33], this can be transformed in reciprocal space into an angle with the $q_{\mathrm{x}}$ axis of $18^{\circ}$, where the $q_{\mathrm{x}}$ axis is parallel to the $\omega / 2 \theta$ axis [34]. This is equal to the angle that the (026) vector makes with the (001) vector perpendicular to the wafer surface. Thus it can be concluded that this streak is perpendicular to the wafer surface. The direction of the crystal lattice strain is thereby confined to the same direction, while the in-plane lattice constant is confined by the underlying substrate [35].

\subsection{Analysis of P-implanted $S i$}

In Fig. 5(a) the rcs of as-implanted samples with $P$ doses of $5 \times 10^{14}$ and $1 \times 10^{15} \mathrm{~cm}^{-2}$ at an energy of $0.5 \mathrm{MeV}$ are shown. The rcs reveal a large tensile strain in the crystal. The implanted $P$ ions and the cascaded $\mathrm{Si}$ atoms at interstitial positions cause tensile strain, reflected by the fringes on the negative side of the main (026) peak. The corresponding dark-field XTEM image (Fig. 5(b)) and the electron diffraction pattern (Fig. 5(c)) show that no amorphization occurred. This is in agreement with previous observations

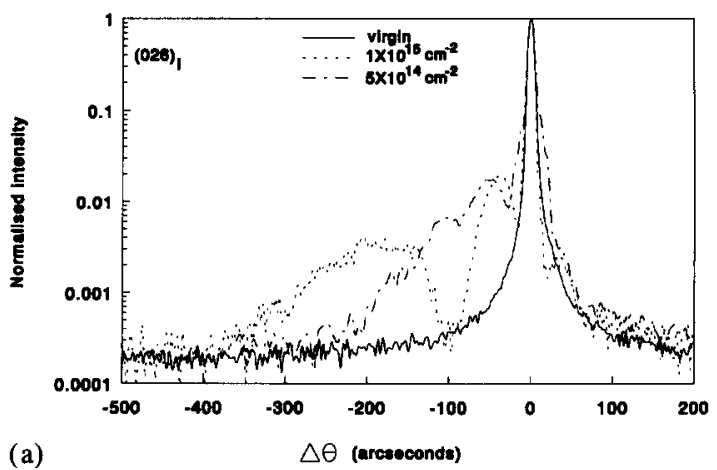

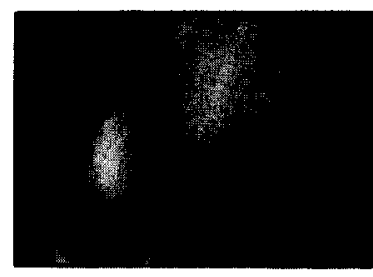

(b)

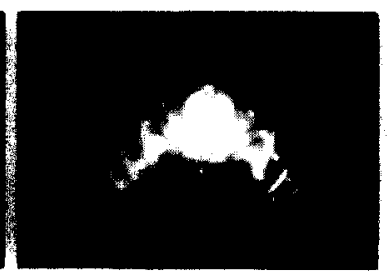

(c)
Fig. 5. Rocking curves of as-implanted $\mathrm{Si}$ at $0.5 \mathrm{MeV}$ with $\mathrm{P}$ along with a virgin reference (a), dark-field XTEM image of the $1 \times 10^{15} \mathrm{~cm}^{-2}$ P-implanted sample (b), and the corresponding electron diffraction pattern (c).

[8], where a threshold value for amorphization of $1 \times 10^{15} \mathrm{P}$ ions $\mathrm{cm}^{-2}$ was found, provided that no selfannealing takes place during implantation. Compared with the lower dose, the strain for the higher dose is about twice as large, as confirmed by computer simulations (not shown here).

After annealing the measured rcs become rather different. Figure 6(a) shows the rcs for three different doses after T-RTA at $1400 \mathrm{~K}$. Differences in implantation energies $(0.5,1$ or $1.5 \mathrm{MeV})$ have no significant influence on the shape in this case. With the lowest doses of $5 \times 10^{13}$ and $7.5 \times 10^{13} \mathrm{~cm}^{-2}$ (not shown here) we obtained the same rc as for the non-implanted sample. Schreutelkamp et al. [36] found that the critical dose for secondary defect formation is about $5 \times 10^{13} \mathrm{~cm}^{-2}$, independent of implantation energy. At $7.5 \times 10^{13} \mathrm{~cm}^{-2}$ a low concentration of secondary defects forms (less than $10^{8}$ dislocations $\mathrm{cm}^{-2}$ ) which is apparently not detectable with XRD. Furthermore, the strain due to the substitutional dopant atoms is below the theoretical detection limit of the XRD technique as shown in Section 3.

At higher implanted doses, more damage sufficient to form secondary defects is present $[8,36]$. During $T$ RTA, the damage clusters coalesce to produce secondary defects in the form of dislocation loops. These loops are located at a depth of approximately $0.6 \mu \mathrm{m}$, corresponding to the implanted projected range, as shown by the bright-field XTEM image in Fig. 6(b). The dislocation loops cause the so-called Huang diffuse 


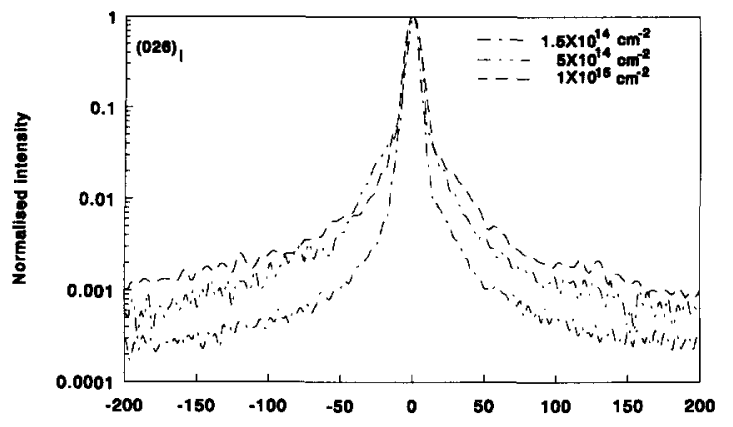

(a)

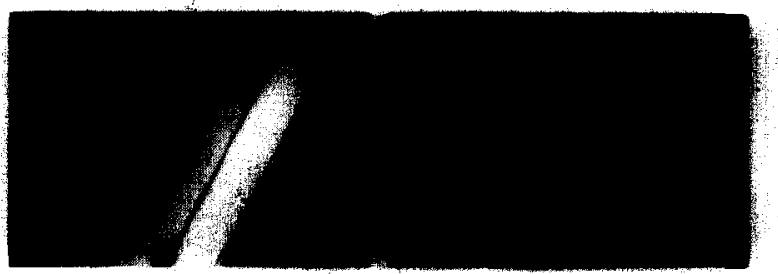

(b)

(c)

Fig. 6. Rocking curves of megaelectronvolt P-implanted Si of various doses, after a T-RTA step at $1400 \mathrm{~K}$ (a), bright-field XTEM image of the $1 \times 10^{15} \mathrm{~cm}^{-2}$ P-implanted sample (b), and the corresponding electron diffraction pattern (c).

scattering [37-39], visible in the rcs of Fig. 6(a) as the increased intensity of tails on both sides of the main peak. The Huang scattering dominates and no fringes are visible originating from the substitutional dopants. The corresponding electron diffraction pattern in Fig. 6(c) also reveals a diffuse intensity, originating from the dislocation loops.

Figure 7 shows rcs of $1.5 \mathrm{MeV}$ P-implanted samples with a low dose of $5 \times 10^{13} \mathrm{~cm}^{-2}$. The upper curve belongs to the non-annealed sample. The large fringes, mainly on the negative side, indicate extensive lattice expansion due to the abundance of interstitial $\mathrm{Si}$ and $\mathrm{P}$ atoms. The other curves were taken on samples subjected to T-RTA at successively increasing temperatures. The samples with peak temperatures of 1200 and $1300 \mathrm{~K}$ still contain decreasingly small fringes on

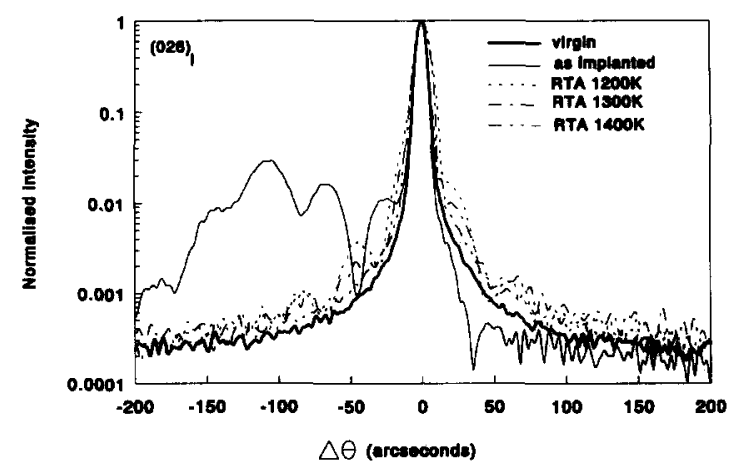

Fig. 7. Rocking curves of $1.5 \times 10^{14} \mathrm{~cm}^{-2} \mathrm{P}$-implanted $\mathrm{Si}$ at $1 \mathrm{MeV}$ $v s$. temperature of the T-RTA step. both sides of the main peak, reflecting the decrease in defect concentration with temperature. No significant further difference could be obtained on any of the wafers annealed at and above $1400 \mathrm{~K}$. The rcs are the same as for the non-implanted sample, indicating complete activation of the implanted $P$ dose. This result is in agreement with our sheet resistivity measurements and electrical lifetime profiling [2]. No remaining implantation damage is expected for these low doses, as explained before.

At a peak temperature of $1200 \mathrm{~K}(300 \mathrm{~ms})$ the damage anneal is incomplete according to Fig. 7. A better result can be achieved with a higher thermal budget, i.e. by increasing the thermal energy supplied to the specimen. In Fig. 7 this was done by raising the peak temperature in the T-RTA step. Another possibility is to extend the anneal time. Complete damage annealing was obtained for example at $1200 \mathrm{~K}$ after $1 \mathrm{~s}$.

\subsection{Analysis of B-implanted $\mathrm{Si}$}

Rcs of B-implanted $\mathrm{Si}$ at an energy of $1 \mathrm{MeV}$ are shown in Fig. 8(a). All samples underwent T-RTA at $1475 \mathrm{~K}$. The doses were varied to analyze the detectability of strain vs. implanted dose by the HR-XRD technique. The measured difference for the lowest doses of $5 \times 10^{13}$ and $1 \times 10^{14} \mathrm{~cm}^{-2}$ with the non-implanted (virgin) sample is negligibly small. This indicates that the strain due to the substitutional B falls below the detection limit of HR-XRD. No increased intensity is detected on the negative side for the dose of $5 \times 10^{13} \mathrm{~cm}^{-2}$ because no secondary defects are present, as confirmed by XTEM in Fig. 8(b). For the dose of $1 \times 10^{14} \mathrm{~cm}^{-2}$, the slightly increased intensity on the negative side corresponds to a very small defect density of about $2 \times 10^{18} \mathrm{~cm}^{-2}$ according to Fig. $8(\mathrm{c})$. This result is in agreement with previous observations [40].

The rc of the sample implanted with a $B$ dose of $5 \times 10^{14} \mathrm{~cm}^{-2}$ contains small fringes on the positive side as well as a small increase in the tail intensity. The XTEM image for this dose in Fig. 8(d) reveals circular dislocation loops with a density of about $10^{9} \mathrm{~cm}^{-2}$. They are located at a depth of about $1.7 \mu \mathrm{m}$, corresponding to the projected range. The number of $\mathrm{Si}$ atoms displaced exceeded the critical number for secondary defect formation [36]. This circular form of dislocation loops was expected for the peak anneal

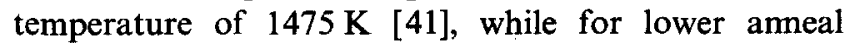
temperatures rod-like defects elongated along $\langle 110\rangle$ directions would be obtained [36].

The fringes on the positive side in Fig. 8(a) are much more apparent than for $P$ at the same implanted dose owing to the smaller atomic size of B compared with $P$. Determination of the doping profile of $B$ from these fringes by computer simulations will be affected by diffuse scattering from the dislocations. 


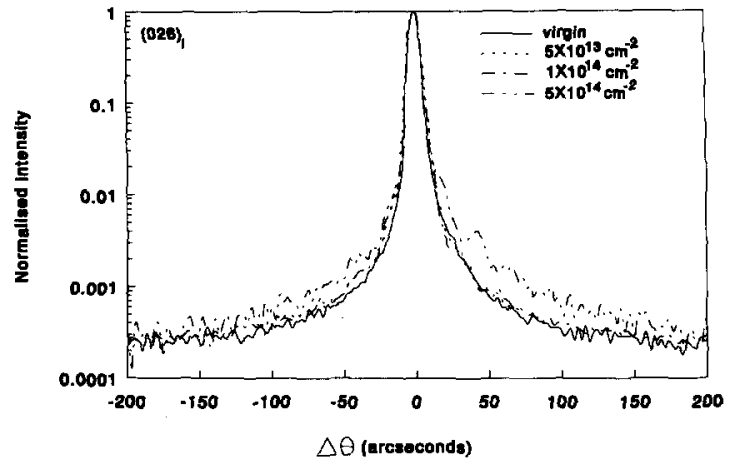

(a)

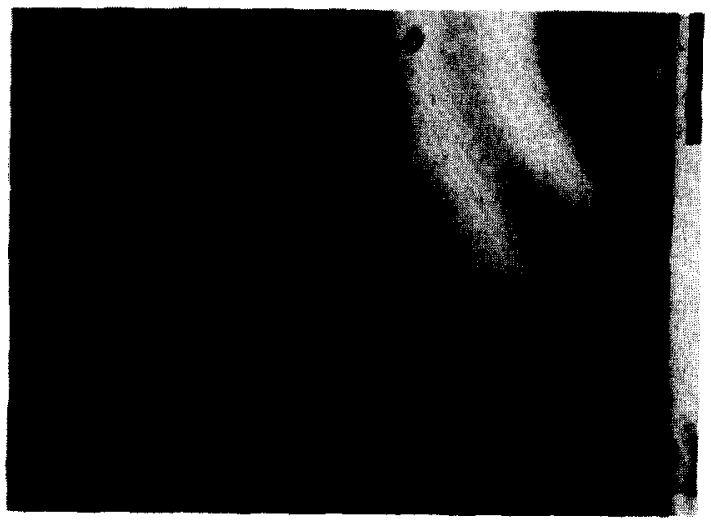

(c)

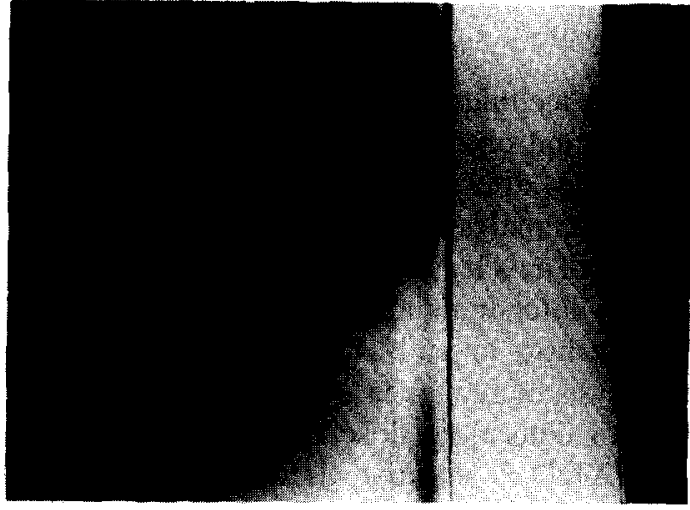

(b)

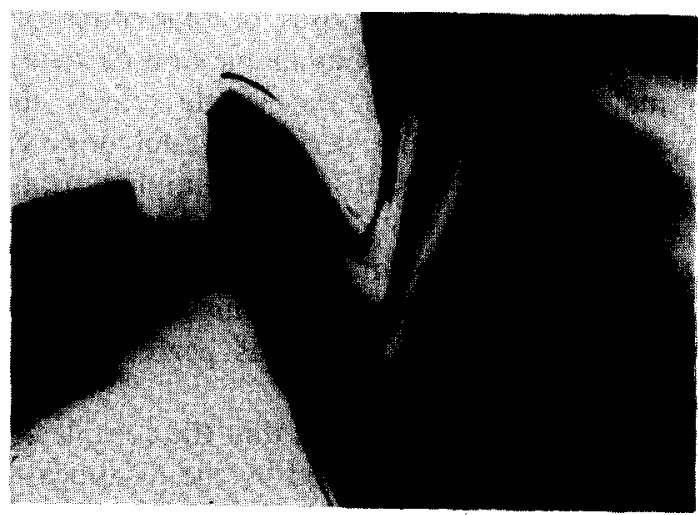

(d)

Fig. 8. Rocking curves of B-implanted Si at $1 \mathrm{MeV}$ measured after a T-RTA step at $1475 \mathrm{~K}$ (a), bright-field XTEM images of the $5 \times 10^{13} \mathrm{~cm}^{-2}$ (b), $1 \times 10^{14} \mathrm{~cm}^{-2}$ (c) and $5 \times 10^{14} \mathrm{~cm}^{-2}$ (d) samples.

To optimize the T-RTA of low dose B-implanted Si, systematic rc analysis was carried out on samples asimplanted and annealed at several peak temperatures. At the same dose of $5 \times 10^{13} \mathrm{~cm}^{-2}$ the fringes in $\mathrm{B}$ implanted samples, compared with $P$, are much closer to the main peak before annealing. This indicates that the lattice damage caused by $\mathbf{B}$ implantation is much smaller, corresponding to the lower dopant ion mass. The rc of the T-RTA samples processed below $1375 \mathrm{~K}$ is identical with that of non-implanted Si. This coincidence is the result of two opposing changes in the lattice constant. The tensile strain originating from the interstitial $\mathrm{B}$ and $\mathrm{Si}$ atoms and the compressive strain from the substitutional $B$ atoms seem to compensate for each other. The samples annealed at $1375 \mathrm{~K}$ and above have very small fringes right from the main peak. This is a consequence of the compressive strain arising from the substitutional $\mathrm{B}$ atoms, as already indicated in Fig. 3 for a $5 \times 10^{14} \mathrm{~cm}^{-2}$ implanted sample. Thus a minimum peak anneal temperature of $1375 \mathrm{~K}$ is required for the T-RTA to annihilate the implantation damage.

Figure 9 is a comparison between the effect of a conventional $30 \mathrm{~min}$ furnace anneal at $1200 \mathrm{~K}$ and

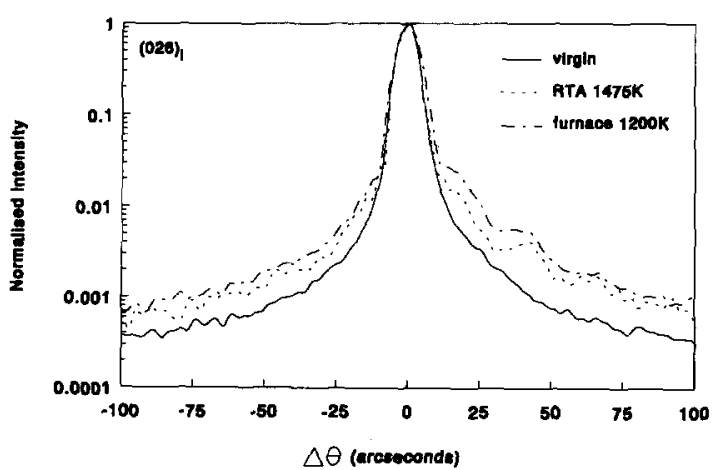

Fig. 9. Comparison between a conventional $30 \mathrm{~min}$ furnace anneal at $1200 \mathrm{~K}$ and the T-RTA step at $1475 \mathrm{~K}$ of $5 \times 10^{14} \mathrm{~cm}^{-2} \mathrm{~B}$-implanted $\mathrm{Si}$ at $1 \mathrm{MeV}$.

that of a T-RTA step at $1475 \mathrm{~K}$ on $5 \times 10^{14} \mathrm{~cm}^{-2} \mathrm{~B}$ implanted samples. The difference between the respective $\mathrm{rcs}$ is the larger B-profile broadening obtained during the furnace anneal, in agreement with earlier spreading resistance profiling results [3]. At the same time the considerably higher diffuse scattering in the tails of the rc of the furnace annealed sample can be ascribed to a larger dislocation density. 


\section{Conclusions}

The HR-XRD technique proves to be a very useful aid for the study and optimization of post-implantation damage annealing after high-energy, low-dose ionimplantation. The analysis is based on the distinction between positive (tensile) and negative (compressive) strain caused by intersitials and substitutional dopants smaller than the host crystal atoms respectively. By a qualitative analysis of rcs taken on B- and P-implanted $\mathrm{Si}$ the minimum detectable dose could be determined for which HR-XRD is sensitive. If the dopants reside at substitutional lattice sites this is about $2 \times 10^{14}$ and $8 \times 10^{13} \mathrm{~cm}^{-2}$ for $P$ and $B$ respectively. In the case of high-energy implanted doses of this order of magnitude, HR-XRD is the only available non-destructive technique for analysis of the subtle effects in the development of crystalline perfection and doping profile distribution as a result of post-implantation annealing.

The best resolution in implantation damage studies is obtainable with the reflection $(026)_{1}$, this being most sensitive to lattice parameter variations perpendicular to the wafer surface.

The qualitative analysis has shown that the completeness of damage annealing is signaled by the disappearance of interference structures on the low-angle side of the rc. The minimum peak temperature $T_{\mathrm{p}}$ for the T-RTA method needed for complete damage annealing could be determined. For $\mathrm{P}$ implants with energies ranging from 0.5 to $1.5 \mathrm{MeV}$ in $\mathrm{Si}$, it was $1400 \mathrm{~K}$ for all doses considered, while for B the minimum peak temperature needed was about $1375 \mathrm{~K}$. Different ramp rates or duration at the peak temperature result in different thermal budgets. The optimum anneal temperature obviously depends on the actual anneal time.

HR-XRD provides cumulative information on the change in lattice constant in implanted specimens. This is a shortcoming of this analysis and requires the use of complementary techniques such as RBS, or the destructive XTEM, SIMS and electrical evaluations. The main issue in further research remains the separation of strain contributions related to crystalline disorder from that caused by substitutional dopants. A quantitative analysis of damage and doping profiles is possible in principle but not straightforward. Related attempts will be reported in a following paper.

\section{Acknowledgments}

This work has been carried out with the financial support of the Dutch Foundation of Fundamental Research on Matters, FOM. The assistance of $\mathbf{M}$. Weusthof, A. Kooy and G. Boom of MESA/UT with sample preparation and Dr. L. Tóth of $\mathrm{CMO} / \mathrm{UT}$ with XTEM analysis is acknowledged.

\section{References}

1 R. C. M. Wijburg, G. J. Hemink, J. Middelhoek, H. Wallinga and T. J. Mouthaan, IEEE Trans. Electron Devices, 38 (1991) 111.

2 I. Bársony, J. L. Heideman, J. Klappe and J. Middelhoek, Jpn. J. Appl. Phys., 30 (1991) 418.

3 I. Bársony, J. L. Heideman, J. Middelhoek and H. Wallinga, Mater. Res. Soc. Symp. Proc., 201 (1991) 277.

4 M. Tamura, N. Natsuaki, Y. Wada and E. Mitani, J. Appl. Phys., 59 (1986) 3417.

5 R. B. Fair, J. Vac. Sci. Technol. A, 4 (1986) 926.

6 R. B. Fair, J. J. Wortman and J. Liu, J. Electrochem. Soc., 131 (1984) 2387.

7 C. Carter, W. Maszara, D. K. Sadans, G. A. Rozgonyi, J. Liu and J. Wortman, Appl. Phys. Lett., 44 (1984) 459.

8 M. Tamura and N. Natsuaki, Jpn. J. Appl. Phys., 25 (1986) L474.

9 M. Tamura, N. Natsuaki, Y. Wada and E. Mitani, Nucl. Instrum. Methods Phys. Res. B, 21 (1987) 438.

10 X. Bao, Q. Guo, M. Hu and D. Feng, J. Appl. Phys., 66 (1989) 1475.

11 A. E. Michel, W. Rausch, P. A. Ronsheim and R. H. Kastl, Appl. Phys. Lett., 50 (1987) 416.

12 Y. M. Kim, G. Q. Lo, D. L. Kwong, A. F. Tasch and S. Novak, Appl. Phys. Lett., 56 (1990) 1254.

13 S. Solmi, F. Baruffaldi and R. Canteri, J. Appl. Phys., 69 (1991) 2135.

14 O. W. Holland, J. Narayan, D. Fathy and S. R. Wilson, J. Appl. Phys., 59 (1986) 905.

15 H. J. Böhm, L. Bernewitz, W. R. Böhm and R. Koepl, IEEE Trans. Electron Devices, 35 (1988) 1616.

16 W. Skorupa, E. Wieser, R. Groetzschel, M. Posselt, H. Buecke, A. Armigliato, A. Garulli, A. Beyer and W. Markgraf, Nucl. Instrum Methods B, 19 (1987) 335.

17 R. Ferretti, W. R. Fahrner and D. Braunig, IEEE Trans. Nucl. Sci., 26 (1979) 4828.

18 K. Yagi, N. Miyamoto and J. Nishizawa, Jpn. J. Appl. Phys., 9 (1970) 246.

19 B. C. Larson and J. F. Barhorst, J. Appl. Phys., $5 I$ (1980) 3181.

20 V. S. Speriosu, B. M. Paine, M. A. Nicolet and H. L. Glass, Appl. Phys. Lett., 40 (1982) 604.

21 O. W. Holland, J. D. Budai and C. W. White, Appl. Phys. Lett., $57(1990) 243$.

22 C. J. Tsai, A. Dommann, M. A. Nicolet and T. Vreeland, Jr., J. Appl. Phys., 69 (1991) 2076.

23 A. Fukuhara and Y. Takano, Acta Crystallogr. A, 33 (1977) 137.

24 P. F. Fewster and C. J. Curling, J. Appl. Phys., 62 (1987) 4154.

25 P. Zaumseil, U. Winter, R. Fabbri, M. Servidori and S. Solmi, Solid State Phenom. A, 94 (1986) 315.

26 T. Tsurshima and H. Tanoue, J. Phys. Soc. Jpn., 31 (1971) 1695.

27 S. M. Sze, Semiconductor Devices, Physics and Technology, Wiley, New York, 1985.

$28 \mathrm{R}$. B. Fair, Concentration profiles of diffused dopants in $\mathrm{Si}$, in F. F. Y. Wang (ed.), Impurity Doping Processes in Si, NorthHolland, New York, 1981, Chapter 7.

29 G. Celotti, D. Nobili and P. Ostoja, J. Mater. Sci., 9 (1974) 821.

30 W. J. Bartels, J. Vac. Sci. Technol. B, 1 (1983) 338.

31 A. Fukuhara and Y. Takano, J. Appl. Crystallogr., 10 (1977) 287. 
32 B. Leroy and C. Plougoven, J. Electrochem. Soc., 127 (1980) 961.

33 A. Iida and K. Kohra, Phys. Status Solidi A, 51 (1979) 533.

34 J. G. E. Klappe, I. Bársony and T. W. Ryan, Mater. Res. Soc. Symp. Proc., 262 (1992) 1121.

35 E. P. EerNisse, Appl. Phys. Lett., 18 (1971) 581.

36 R. J. Schreutelkamp, J. S. Custer, J. R. Liefting, W. X. Lu and F. W. Saris, Mater. Sci. Rep., 6(1991) 17.
37 K. Huang and H. H. Willis, Proc. R. Soc. London, Ser A, 190 (1947) 102.

38 P. Zaumseil, U. Winter, F. Cembali, M. Servidori and Z. Sourek, Phys. Status Solidi A, 100 (1987) 95.

39 K. Lal, Prog. Cryst. Growth Charact., 18 (1989) 227.

40 J. R. Liefting, J. S. Custer and F. W. Saris, Mater. Res. Soc. Symp. Proc, 235 (1992) 179.

41 K. Seshan and J. Washburn, Radiat. Effects, 37 (1978) 147. 